\title{
Randomly coloring simple hypergraphs
}

\author{
Alan Frieze* Páll Melsted \\ Department of Mathematical Sciences, \\ Carnegie Mellon University, \\ Pittsburgh PA15213. \\ Email: alan@random.math.cmu.edu; pmelsted@gmail.com.
}

June 1, 2011

\begin{abstract}
We study the problem of constructing a (near) uniform random proper $q$-coloring of a simple $k$-uniform hypergraph with $n$ vertices and maximum degree $\Delta$. (Proper in that no edge is mono-colored and simple in that two edges have maximum intersection of size one). We show that if for some $\alpha<1$ we have $\Delta \geq n^{\alpha}$ and $q \geq \Delta^{(1+\alpha) / k \alpha}$ then Glauber dynamics will become close to uniform in $O(n \log n)$ time from a random (improper) start. Note that for $k>1+\alpha^{-1}$ we can take $q=o(\Delta)$.
\end{abstract}

\section{Introduction}

Markov Chain Monte Carlo (MCMC) is an important tool in sampling from complex distributions. It has been successfully applied in several areas of Computer Science, most notably for estimating the volume of a convex body [3], [8], [9] and estimating the permanent of a non-negative matrix [7].

Generating a (nearly) random $q$-coloring of a $n$-vertex graph $G=(V, E)$ with maximum degree $\Delta$ is a well-studied problem in Combinatorics [1] and Statistical Physics [10]. Jerrum [6] proved that a simple, popular Markov chain, known as the Glauber dynamics, converges to a random $q$-coloring after $O(n \log n)$ steps, provided $q / \Delta>2$. This led to the challenging problem of determining the smallest value of $q / \Delta$ for which a random $q$-coloring can be generated in time polynomial in $n$. Vigoda [11] gave the first significant improvement over Jerrum's result, reducing the lower bound on $q / \Delta$ to $11 / 6$ by analyzing a different Markov chain. There has been no success in extending Vigoda's approach to smaller values of $q / \Delta$, and it remains the best bound for general graphs. There are by now several papers giving improvements on [11], but in special cases. See Frieze and Vigoda [5] for a recent survey.

\footnotetext{
* Supported in part by NSF grant CCF-0502793.
} 
In this paper we consider the related problem of finding a random coloring of a simple $k$-uniform hypergraph. A $k$-uniform hypergraph $H=(V, E)$ has vertex set $V$ and $E=$ $\left\{e_{1}, e_{2}, \ldots, e_{m}\right\}$ are the edges. Each edge is a $k$-subset of $V$. Hypergraph $H$ is simple if $\left|e_{i} \cap e_{j}\right| \leq 1$ for $i \neq j$. A coloring of $H$ is proper if every edge contains two vertices of a different color. The chromatic number $\chi(H)$ is the smallest number of colors in a proper coloring of $H$. In the case of graphs $k=2$ we have $\chi(G) \leq \Delta+1$ but for hypergraphs $(k \geq 3)$ we have much smaller bounds. For example a simple application of the local lemma implies that $\chi(H)=O\left(\Delta^{1 /(k-1)}\right)$. In fact a recent result of Frieze and Mubayi [4], is that for simple hypergraphs $\chi(H)=O\left((\Delta / \log \Delta)^{1 /(k-1)}\right)$. The proof of [4] is somewhat more involved. It relies on a proof technique called the "nibble". The aim of this short paper is to study randomly coloring simple hypergraphs when there are fewer than $\Delta$ colors available. The goal is to describe an MCMC algorithm for (near) uniform sampling.

Before formally stating our theorem we will define the Glauber dynamics. All of the aforementioned results on coloring graphs (except Vigoda [11]) analyze the Glauber dynamics, which is a simple and popular Markov chain for generating a random $q$-coloring.

Let $\mathcal{Q}$ denote the set of proper $q$-colorings of $H$. For a coloring $X \in \mathcal{Q}$ we define

$$
B(v, X)=\{c \in[q]: \exists e \ni v \text { such that } X(x)=c \text { for all } x \in e \backslash\{v\}\}
$$

be the set of colors unavailable to $v$.

Then let $Q=\{1,2, \ldots, q\}$ and

$$
A(v, X)=Q \backslash B(v, X) .
$$

For technical purposes, the state space of the Glauber dynamics is $\Omega=Q^{V} \supseteq \mathcal{Q}$. From a coloring $X_{t} \in \Omega$, the evolution $X_{t} \rightarrow X_{t+1}$ is defined as follows:

\section{Glauber Dynamics}

(a) Choose $v=v(t)$ uniformly at random from $V$.

(b) Choose color $c=c(t)$ uniformly at random from $A\left(v, X_{t}\right)$. If $A\left(v, X_{t}\right)$ is empty we let $X_{t+1}=X_{t}$.

(c) Define $X_{t+1}$ by

$$
X_{t+1}(u)= \begin{cases}X_{t}(u) & u \neq v \\ c & u=v\end{cases}
$$

We will assume from now on that

$$
q \leq 2 \Delta
$$

If $q>2 \Delta$ then we defer to Jerrum's result [6].

Let $Y$ denote a coloring chosen uniformly at random from $\mathcal{Q}$. We will prove the following:

Theorem 1 Let $H$ be a $k$-uniform simple hypergraph with maximum degree $\Delta$ where $k \geq 3$. Suppose that (1) holds and that

$$
\Delta \geq n^{\alpha}
$$


for some $\alpha$ satisfying

$$
\begin{array}{lr}
\frac{2}{k-2}<\alpha<1 & k \geq 5 \\
\frac{1}{k-1}<\alpha<1 & k=3,4
\end{array}
$$

Suppose that for a sufficiently large constant $K$,

$$
q \geq K \Delta^{(1+\alpha) / \alpha k}
$$

Suppose that the initial coloring $X_{0}$ is chosen randomly from $\Omega$. Then for an arbitrary constant $\delta>0$ we have

$$
d_{T V}\left(X_{t}, Y\right) \leq \delta
$$

for $t \geq t_{\delta}$, where $t_{\delta}=2 n \log (2 n / \delta)$.

Here $d_{T V}$ denotes variational distance i.e. $\max _{S \subseteq \mathcal{Q}}\left|\operatorname{Pr}\left(X_{t} \in S\right)-\operatorname{Pr}(Y \in S)\right|$.

Our assumptions on $\alpha, \Delta$ imply some inequalities that we put here for future reference:

$$
\begin{array}{ll}
q^{k} \gg n \Delta . & \\
q^{k-2} \gg \Delta & \text { for } k \geq 5 . \\
q \gg \Delta^{2 / 3} & \text { for } k=3 \\
q \gg \Delta^{1 / 2} & \text { for } k=4
\end{array}
$$

Here we use $A_{n} \gg B_{n}$ to mean that $A_{n} / B_{n} \rightarrow \infty$ as $n \rightarrow \infty$.

Note that $H$ simple implies that

$$
\Delta \leq \frac{n-1}{k-1}<\frac{n}{k}
$$

Note that we do not claim rapid mixing from an arbitrary start. Indeed, since we are using relatively few colors, it is possible to choose an initial coloring from which there is no Glauber move i.e. we do not claim that the chain is ergodic, see Section 5 for examples of blocked colorings.

The algorithm can be used in a standard way, [6], to compute an approximation to the number of proper colorings of $H$.

We can also prove the following. We can consider Glauber Dynamics as inducing a graph $\Gamma_{\mathcal{Q}}$ on $\mathcal{Q}$ where two colorings are connected by an edge if there is a move taking one to the other. Note that if Glauber can take $X$ to $Y$ in one step, then it can take $Y$ to $X$ in one step.

Corollary 1 The graph $\Gamma_{\mathcal{Q}}$ contains a giant component $\mathcal{Q}_{0}$ of size $(1-o(1))|\mathcal{Q}|$. 


\section{Good and bad colorings}

Let $X \in \Omega$ be a coloring of $V$. For a vertex $v$ and $1 \leq i \leq k-1$ let

$$
E_{v, i, X}=\{e: v \in e \text { and }|\{X(w): w \in e \backslash\{v\}\}|=i\}
$$

be the set of edges $e$ containing $v$ in which $e \backslash\{v\}$ uses exactly $i$ distinct colors under $X$. Let $y_{v, i, X}=\left|E_{v, i, X}\right|$ so that $|B(v, X)| \leq y_{v, 1, X}$ for all $v, X$. Let

$$
\epsilon=\frac{1}{100(2 k)^{k}} .
$$

We divide our analysis into three cases: $k \geq 5, k=3$ and $k=4$.

\section{$2.1 \quad k \geq 5$}

We define the sequence $\boldsymbol{\epsilon}=\epsilon, \epsilon^{2}, \ldots, \epsilon^{k-2}$.

Definition 1 We say that $X$ is $\theta \boldsymbol{\epsilon}$-bad if $\exists v \in V, 1 \leq i \leq k-2$ such that

$$
y_{v, i, X} \geq \theta \mu_{i} \text { where } \mu_{i}=\min \left\{(\epsilon q)^{i}, \frac{\Delta}{(10 k)^{k-2-i} \epsilon q}\right\} \text {. }
$$

Otherwise we say that $X$ is $\theta \boldsymbol{\epsilon}$-good. (If $\theta=1$ then we drop it).

Given this definition, we have

$$
10 k \mu_{i} \leq \mu_{i+1} \leq \epsilon q \mu_{i} \quad \text { for } 1 \leq i \leq k-3 .
$$

It is convenient to define

$$
\mu_{k-1}=\Delta
$$

Note that if $X$ is $\boldsymbol{\epsilon}$-good then $|A(v, X)| \geq(1-\epsilon) q$ for all $v \in V$.

In this section we will show that almost all colorings of $\Omega$ are $\boldsymbol{\epsilon}$-good and almost all colorings in $\mathcal{Q}$ are $\boldsymbol{\epsilon}$-good. Consider a random coloring $X \in \Omega$. We first estimate the probability that it is properly colored. We use the local lemma.

Fix an edge $e \in H$. Then using $\operatorname{Pr}_{\Omega}$ to indicate that the random choice is from $\Omega$,

$$
p=\operatorname{Pr}_{\Omega}(e \text { is not properly colored by } X)=\frac{1}{q^{k-1}} .
$$

Now consider a dependency graph, in the context of the local lemma. The events are $\mathcal{B}_{e}=\{e$ is not properly colored) $\} . \quad \mathcal{B}_{e}$ and $\mathcal{B}_{f}$ are indpendent if $e \cap f=\emptyset$. Thus the maximum degree $\Delta_{1}$ in the dependency graph is bounded by $k \Delta$. Then

$$
4 \Delta_{1} p \leq 4 k \Delta p \leq \frac{4 k \Delta}{K^{k-1} \Delta^{(k-1)(1+\alpha) /(\alpha k)}}=\frac{4 k}{K^{k-1} \Delta^{(k-1-\alpha) /(\alpha k)}}<1 .
$$


So, by the local lemma, if $m \leq \Delta n / k$ is the number of edges in $H$,

$$
\operatorname{Pr}_{\Omega}(X \text { is proper }) \geq(1-2 p)^{m} \geq(1-2 p)^{\Delta n / k} \geq e^{-2 p \Delta n / k(1-2 p)} \geq e^{-3 \Delta n /\left(k q^{k-1}\right)} .
$$

For the third inequality we use $(1-x) \geq e^{-x /(1-x)}$ whenever $0<x<1$.

Given this, we consider the probability that there is a bad vertex. For a fixed vertex $v$ and an edge $e$ containing $v$ the probability that $e \in E_{v, i, X}$ is at most $\left(\begin{array}{c}k-1 \\ i\end{array}\right)\left(\frac{i}{q}\right)^{k-1-i}$. Indeed, there will be a set of $i$ vertices $S$, for which there are $\left(\begin{array}{c}k-1 \\ i\end{array}\right)$ possibilities such that the remaining $k-1-i$ vertices have their colors all used by $S$. The probability of this being at most $\left(\frac{i}{q}\right)^{k-1-i}$. Thus the value $y_{v, i, X}$ has distribution dominated by the binomial $\operatorname{Bin}\left(\Delta,\left(\begin{array}{c}k-1 \\ i\end{array}\right)\left(\frac{i}{q}\right)^{k-1-i}\right)$.

So, from Chernoff bound:

$$
\operatorname{Pr}(B(n, p) \geq \lambda n p) \leq\left(\frac{e}{\lambda}\right)^{\lambda n p}
$$

we see that

$$
\begin{aligned}
\operatorname{Pr}\left(y_{v, i, X} \geq \mu_{i}\right) & \leq\left(\frac{e\left(\begin{array}{c}
k-1 \\
i
\end{array}\right)(i / q)^{k-1-i} \Delta}{\mu_{i}}\right)^{\mu_{i}} \\
& \leq\left(\frac{e(2 k)^{k} \Delta}{\min \left\{\epsilon^{i} q^{k-1}, \epsilon^{-1}(q /(10 k))^{k-2-i} \Delta\right\}}\right)^{\mu_{i}} \\
& \leq e^{-\mu_{i}} \\
& \leq e^{-\epsilon \min \{q, \Delta / q\}}
\end{aligned}
$$

for $i=1,2, \ldots, k-2$, since $\mu_{i} \geq \mu_{1} \geq \epsilon \min \{q, \Delta / q\}$. (We use (6) and our definition of $\epsilon$ to go from the second to the third line).

It follows that if $X$ is chosen randomly from $\Omega$ then

$$
\operatorname{Pr}_{\Omega}(X \text { is } \boldsymbol{\epsilon}-\text { bad }) \leq k n e^{-\epsilon \min \{q, \Delta / q\}} .
$$

So, using $\operatorname{Pr}_{\mathcal{Q}}$ to indicate the random choice is from $\mathcal{Q}$, and using (13),

$$
\begin{aligned}
& \operatorname{Pr}_{\mathcal{Q}}(X \text { is } \boldsymbol{\epsilon}-b a d)=\operatorname{Pr}_{\Omega}(X \text { is } \boldsymbol{\epsilon}-\text { bad } \mid X \text { is a proper coloring }) \\
& \leq k n \exp \left\{-\epsilon \min \{q, \Delta / q\}+\frac{3 n \Delta}{k q^{k-1}}\right\} \\
& \leq e^{-\epsilon \min \{q, \Delta / q\} / 2}=o(1)
\end{aligned}
$$

after using (5) and (6).

Thus whp, a random proper or improper $q$-coloring of $H$ is $\boldsymbol{\epsilon}$-good.

Remark 1 We observe that if $q^{k-1} / n \Delta \rightarrow \infty$ then a random coloring (from $\Omega$ ) is proper whp, in which case we won't need to use the local lemma. 
Remark 2 It is reasonable to conjecture that $\operatorname{Pr}_{\mathcal{Q}}(X$ is $\boldsymbol{\epsilon}-$ bad $)$ is not much larger than $\operatorname{Pr}_{\Omega}(X$ is $\boldsymbol{\epsilon}-$ bad $)$. Using the Local Lemma inflates the first proability by too much, seemingly. A better estimate of the first probability would allow us to consider smaller $q$.

\section{$2.2 k=3$}

In this case we let $\mu_{1}=\epsilon q . y_{v, 1, X}$ has distribution dominated by $\operatorname{Bin}(\Delta, 1 / q)$ and so

$$
\operatorname{Pr}\left(y_{v, 1, X} \geq \mu_{1}\right) \leq\left(\frac{e \Delta}{q \mu_{1}}\right)^{\mu_{1}} \leq e^{-q}
$$

since $q \gg \Delta^{2 / 3}$ here, see (7). So

$$
\operatorname{Pr}_{\mathcal{Q}}(X \text { is } \boldsymbol{\epsilon}-b a d) \leq 3 n \exp \left\{-q+\frac{n \Delta}{q^{2}}\right\}=o(1)
$$

using (5).

\section{$2.3 k=4$}

In this case we let $\mu_{1}=\epsilon q$ and $\mu_{2}=10 k \mu_{1}$. $y_{v, 1, X}$ has distribution dominated by $\operatorname{Bin}\left(\Delta, 3 / q^{2}\right)$ and $y_{v, 2, X}$ has distribution dominated by $\operatorname{Bin}(\Delta, 3 / q)$ so

$$
\begin{aligned}
& \operatorname{Pr}\left(y_{v, 1, X} \geq \mu_{1}\right) \leq\left(\frac{3 e \Delta}{q^{2} \mu_{1}}\right)^{\mu_{1}} \leq e^{-q} \\
& \operatorname{Pr}\left(y_{v, 2, X} \geq \mu_{2}\right) \leq\left(\frac{3 e \Delta}{q \mu_{2}}\right)^{\mu_{2}} \leq e^{-q}
\end{aligned}
$$

since $q \gg \Delta^{1 / 2}$ here, see (8). So

$$
\operatorname{Pr}_{\mathcal{Q}}(X \text { is } \boldsymbol{\epsilon}-b a d) \leq 4 n \exp \left\{-q+\frac{3 n \Delta}{4 q^{3}}\right\}=o(1)
$$

using (5).

\section{Persistence of goodness}

We once again divide our analysis into three cases: $k \geq 5, k=3$ and $k=4$.

\section{$3.1 k \geq 5$}

We show first that 


\section{Lemma 1}

$$
\operatorname{Pr}\left(X_{t} \text { is } 2 \boldsymbol{\epsilon}-\operatorname{good} \text { for } t \leq t_{0} \mid X_{0} \text { is } \boldsymbol{\epsilon}-\text { good }\right) \geq 1-2^{-\mu_{1} / 2}
$$

where

$$
t_{0}=\frac{n}{4 k^{2} e}
$$

Proof For vertices $x, y \in V$ that share an edge, let $e(x, y)$ be that edge.

Observe that if $X_{t}$ is $2 \epsilon$-good and $x \in V$ then

$$
\operatorname{Pr}\left(y_{x, i, t+1}-y_{x, i, t}=1 \mid X_{t}\right) \leq \frac{(k-2)^{2} y_{x, i+1, t}}{(1-2 \epsilon) q n}+\frac{k y_{x, i-1, t}}{n}
$$

for $1 \leq i \leq k-2$. (Here $\left.y_{x, 0, t}=0\right)$.

Indeed, we have $y_{x, i, t+1}-y_{x, i, t}=1$ only if with $v=v(t)$ we have (i) $e(v, x) \in E_{x, i+1, X_{t}}$ and (ii) $X_{t}(v)$ is a color used once on $e(v, x) \backslash\{x\}$ and (iii) $c(t)$ is used on $e(v, x) \backslash\{x, v\}$ or we have (ia) $e(v, x) \in E_{x, i-1, X_{t}}$.

Now

$$
\begin{aligned}
\operatorname{Pr}((i),(i i),(i i i))=\operatorname{Pr}((i)) \cdot \operatorname{Pr}((i i) \mid(i)) \cdot \operatorname{Pr}((i i i) \mid(i),(i i)) \leq \\
\frac{(k-1) y_{x, i+1, t}}{n} \cdot \frac{i}{k-1} \cdot \frac{i}{(1-2 \epsilon) q} \leq \frac{(k-2)^{2} y_{x, i+1, t}}{(1-2 \epsilon) q n}
\end{aligned}
$$

and

$$
\operatorname{Pr}((i a)) \leq \frac{(k-1) y_{x, i-1, t}}{n}
$$

We consider the following sequence of events for $1 \leq i \leq k-2$ :

$$
\mathcal{B}_{i}(t)=\left\{\exists s \leq t, v \in V: X_{\tau} \text { is } 2 \epsilon-\operatorname{good} \text { for } \tau<s \text { and } y_{v, i, s} \geq y_{v, i, 0}+\mu_{i}\right\}
$$

Let $\mathcal{B}(t)=\bigcup_{i=1}^{k-2} \mathcal{B}_{i}(t)$ and note that if $\neg \mathcal{B}(t)$ then $X_{t}$ is $2 \boldsymbol{\epsilon}$-good.

Now $X_{0}$ is $\boldsymbol{\epsilon}$-good and so (18) implies that so long as $X_{\tau}$ is $2 \boldsymbol{\epsilon}$-good for $\tau<s \leq t$, we have

$$
\begin{array}{r}
y_{v, k-2, s} \leq y_{v, k-2,0}+\operatorname{Bin}\left(s, \frac{(k-2)^{2} y_{v, k-1, t}}{(1-2 \epsilon) q n}+\frac{k y_{v, k-3, t}}{n}\right) \\
\leq y_{v, k-2,0}+\operatorname{Bin}\left(s, \frac{k^{2}}{n}\left(\frac{\Delta}{q}+\frac{2 \mu_{k-3}}{k}\right)\right) .
\end{array}
$$

The probability in the first binomial above is the RHS of (18) and in the second we bound $y_{v, k-1, t}$ by $\Delta$ and $y_{v, k-3, t}$ by $2 \mu_{k-3}$. Then note that $y_{v, k-2, i+1}$ is $y_{v, k-2, i}+1$ with at most this probability. So, on using (14),

$$
\begin{aligned}
\operatorname{Pr}\left(\mathcal{B}_{k-2}(t)\right) & \leq \operatorname{tn}\left(\frac{2 e t k^{2}}{n}\left(\frac{\Delta}{\mu_{k-2} q}+\frac{2 \mu_{k-3}}{k \mu_{k-2}}\right)\right)^{\mu_{k-2}} \\
& \leq \operatorname{tn}\left(\frac{2 e t k^{2}}{n}\left(\frac{\Delta}{\epsilon^{k-2} q^{k-1}}+\epsilon+\frac{1}{5 k^{2}}\right)\right)^{\mu_{k-2}} \leq t n\left(\frac{2 e t k^{2}}{n}\right)^{\mu_{k-2}} \leq \frac{t_{0} n}{2^{\mu_{k-2}}} .
\end{aligned}
$$


We have dealt with the minimum in the definition of $\mu_{k-2}$ by using the inequality $\frac{A}{\min X, Y} \leq$ $\frac{A}{X}+\frac{A}{Y}$. The reader will observe that we have not shown that $t_{0} \geq \mu_{k-2}$. We do not claim this and when $t_{0}<\mu_{k-2}$ we can replace the RHS of (20) by zero.

For $i<k-2,(18)$ implies that so long as $X_{\tau}$ is $2 \epsilon$-good for $\tau<s \leq t$ and $\bigcup_{j=i+1}^{k-2} \mathcal{B}_{j}(t)$ does not occur, we have $y_{v, i, s} \leq y_{v, i, 0}+\operatorname{Bin}(s, p)$ where

$$
p=\frac{2(k-2)^{2} \mu_{i+1}}{(1-2 \epsilon) q n}+\frac{2 k \mu_{i-1}}{n} .
$$

So, on using (10),

$\operatorname{Pr}\left(\mathcal{B}_{i}(t) \mid \neg \bigcup_{j=i+1}^{k-2} \mathcal{B}_{j}(t)\right) \leq t_{0} n\left(\frac{2 e(k-2)^{2} \mu_{i+1} t}{(1-2 \epsilon) q n \mu_{i}}+\frac{2 k e \mu_{i-1} t}{n \mu_{i}}\right)^{\mu_{i}} \leq t_{0} n\left(\frac{2 k^{2} e t}{n}\right)^{\mu_{i}} \leq \frac{t_{0} n}{2^{\mu_{i}}}$.

Equation (16) and the lemma follows from (20) and (21).

We show next that

\section{Lemma 2}

$$
\operatorname{Pr}\left(X_{t_{0}} \text { is } \epsilon-\operatorname{good} \mid X_{0} \text { is } \boldsymbol{\epsilon}-\text { good }\right) \geq 1-e^{-c \mu_{1}} \quad \text { for some } c>0 .
$$

Proof For this we use the fact that if $X_{t}$ is $2 \epsilon$-good and $x \in V$ then

$$
\operatorname{Pr}\left(y_{x, i, t+1}-y_{x, i, t}=-1 \mid X_{t}\right) \geq \frac{y_{x, i, t}}{n}
$$

for $1 \leq i \leq k-2$.

Indeed, we have $y_{x, i, t+1}-y_{x, i, t}=-1$ if (i) $e(v, x) \in E_{x, i, X_{t}}$, (ii) $X_{t}(v)$ is used more than once on $e(v, x) \backslash\{x\}$ and (iii) $c(t)$ is a color not used $e(v, x) \backslash\{x, v\}$. Now

$$
\begin{aligned}
& \operatorname{Pr}((i),(i i),(i i i))=\operatorname{Pr}((i)) \cdot \operatorname{Pr}((i i) \mid(i)) \cdot \operatorname{Pr}((i i i) \mid(i),(i i)) \geq \\
& \frac{(k-1) y_{x, i, t}}{n} \cdot \frac{k-i}{k} \cdot \frac{q-i}{q} \geq \frac{2(k-1)(q-i) y_{x, i, t}}{k q n} \geq \frac{y_{x, i, t}}{n} .
\end{aligned}
$$

We couple $y_{x, i, t}$ with a biased random walk $Y_{t}, t \geq 0$ on $\{0,1,2, \ldots$,$\} . Here Y_{0}=\mu_{i}$ and

$$
Y_{t+1}= \begin{cases}Y_{t}+1 & \text { Probability } \frac{2(k-2)^{2} \mu_{i+1}}{(1-2 \epsilon) q n}+\frac{2 k \mu_{i-1}}{n} \\ Y_{t}-1 & \text { Probability } \frac{Y_{t}}{n} \\ Y_{t} & \text { Probability } 1-\frac{2(k-2)^{2} \mu_{i+1}}{(1-2 \epsilon) q n}-\frac{2 k \mu_{i-1}}{n}-\frac{Y_{t}}{n}\end{cases}
$$

If $\mathcal{B}(t)$ does not occur then $Y_{t}$ has no lower a chance of increasing by one than $y_{x, i, t}$ and when $Y_{t}=y_{i, x, t}$ it has no greater a chance of decreasing by one. We can therefore, conditional on $\neg \mathcal{B}(t)$, couple $y_{x, i, t}, Y_{t}$ so that $y_{x, i, t} \leq Y_{t}$ always. We can therefore prove (22) by proving

$$
\operatorname{Pr}\left(Y_{t_{0}}>\mu_{i}\right) \leq e^{-c \mu_{i}}
$$


Let $I=\left\{t \leq t_{0}: Y_{t} \geq \mu_{i} / 2\right\}$ and $J=\left\{t \leq t_{0}: Y_{t+1} \neq Y_{t}\right\}$. If $I=\emptyset$ then $Y_{t_{0}}<\mu_{1} / 2$. Furthermore, if $t \in I$ then (i) $\operatorname{Pr}(t \in J) \geq \mu_{i} /(2 n)$ and (ii) $\operatorname{Pr}\left(Y_{t+1}=Y_{t}-1 \mid Y_{t+1} \neq Y_{t}\right) \geq$ $\frac{2}{3}$. To verify (ii) use (10) to show that

$$
\frac{2 n}{\mu_{i}} \cdot\left(\frac{2 k^{2} \mu_{i+1}}{(1-2 \epsilon) q n}+\frac{2 k \mu_{i-1}}{n}\right) \leq \frac{4 \epsilon k^{2}}{1-2 \epsilon}+\frac{4 k}{10 k} \leq \frac{1}{2} .
$$

Now consider two cases:

Case 1: $I=\left[t_{0}\right]$ : It follows from (i), (ii) above and the Chernoff bounds that

$$
\operatorname{Pr}\left(|J| \leq \frac{\mu_{i} t_{0}}{4 n}\right) \leq e^{-c_{1} \mu_{i}} \quad \text { for some } c_{1}>0 .
$$

And

$$
\operatorname{Pr}\left(\sum_{t \in J}\left(Y_{t+1}-Y_{t}\right) \geq-|J| / 6|| J \mid \geq \frac{\mu_{i} t_{0}}{4 n}\right) \leq e^{-c_{2} \mu_{i}} \quad \text { for some } c_{2}>0 .
$$

But $Y_{t_{0}} \leq \mu_{i}+\sum_{t \in J}\left(Y_{t+1}-Y_{t}\right)$ and so (25) is verified for this case.

Case 2: $I \neq\left[t_{0}\right]$ : Let $t_{1}=\max \{t: t \notin I\}$ and let $J_{1}=\left\{t \in J: t>t_{1}\right\}$. If $\left|J_{1}\right|<\mu_{i} / 2$ then $Y_{t_{0}} \leq \frac{\mu_{1}}{2}+\left|J_{1}\right|<\mu_{1}$. So assume that $\left|J_{1}\right| \geq \mu_{i} / 2$. But then with probability $1-e^{-c_{3} \mu_{i}}$ we find that $\sum_{t \in J_{1}}\left(Y_{t+1}-Y_{t}\right)<0$ and $(25)$ is verified for this case too.

This completes the proof of (25), and hence (22) and the lemma.

\section{$3.2 k=3$}

Going back to (19) we see that

$$
y_{v, 1, s} \leq y_{v, 1,0}+\operatorname{Bin}\left(s, \frac{k^{2} \Delta}{n q}\right)
$$

And then (20) becomes

$$
\operatorname{Pr}\left(\mathcal{B}_{1}(t)\right) \leq t n\left(\frac{18 e \Delta t}{n q \mu_{1}}\right)^{\mu_{1}} \ll 2^{-\mu_{1} / 2}
$$

for $t \leq t_{0}$ of (17).

We then define $Y_{t}$ as in $(24)$ and (see (26)) compute $\frac{2 n}{\mu_{1}} \cdot \frac{2 \Delta}{(1-2 \epsilon) q n}=\frac{4 \Delta}{(1-2 \epsilon) \epsilon q^{2}} \ll 1$. We then argue as in the case $k \geq 5$.

\section{$3.3 k=4$}

Going back to (20) we see that

$$
\operatorname{Pr}\left(\mathcal{B}_{2}(t)\right) \leq \operatorname{tn}\left(\frac{32 e t}{n}\left(\frac{\Delta}{\epsilon q^{2}}+\frac{2}{4}\right)\right)^{\mu_{2}} \ll 2^{-\mu_{2} / 2} .
$$


for $t \leq t_{0}$ of (17).

Going back to (21) we see that

$$
\operatorname{Pr}\left(\mathcal{B}_{1}(t) \mid \neg \mathcal{B}_{2}(t)\right) \leq t_{0} n\left(\frac{8 e t}{(1-2 \epsilon) q n}\right)^{\mu_{1}} \ll 2^{-\mu_{1} / 2}
$$

for $t \leq t_{0}$ of (17).

We then define $Y_{t}$ as in (24) and (see (26)) compute $\frac{2 n}{\mu_{1}} \cdot \frac{8 \mu_{2}}{(1-2 \epsilon) q n}=\frac{160 k}{(1-2 \epsilon) \epsilon q} \ll 1$ (remember that $\mu_{2}=10 k \mu_{1}$ here) and $\frac{2 n}{\mu_{2}}\left(\frac{8 \Delta}{(1-2 \epsilon) q n}+\frac{2 k \mu_{1}}{n}\right) \leq \frac{1}{2}$. We then argue as in the case $k \geq 5$.

\section{Coupling Argument}

Now consider a pair $X, Y$ of copies of our Glauber chain. Let

$$
h\left(X_{t}, Y_{t}\right)=\left|\left\{v \in V: X_{t}(v) \neq Y_{t}(v)\right\}\right|
$$

be the Hamming distance between $X_{t}, Y_{t}$. We use describe a simple coupling between the chains and show that

$$
\mathbf{E}\left(h\left(X_{t+1}, Y_{t+1}\right) \mid X_{t}, Y_{t}\right) \leq\left(1-\frac{1}{2 n}\right) h\left(X_{t}, Y_{t}\right)
$$

if $X_{t}, Y_{t}$ are both $2 \epsilon$-good.

Our coupling is the same as that used by Jerrum [6]. The choice of vertex $v(t)$ will be the same in both chains. We maximally couple the choice of color in each chain. Then, with $v=v(t)$,

$$
\operatorname{Pr}\left(X_{t+1}(v) \neq Y_{t+1}(v) \mid X_{t}, Y_{t}\right) \leq(k-1) \frac{\left|E_{v, 1, X_{t}}\right|+\left|E_{v, 1, Y_{t}}\right|}{(1-2 \epsilon) q} .
$$

Hence, assuming that $X_{t}, Y_{t}$ are both $2 \epsilon$-good for $1 \leq t \leq t_{0}$ we see that

$$
\begin{aligned}
\mathbf{E}\left(h\left(X_{t+1}, Y_{t+1}\right) \mid X_{t}, Y_{t}\right)= & \sum_{v \in V} \operatorname{Pr}\left(X_{t+1}(v) \neq Y_{t+1}(v)\right) \\
= & \sum_{w \in V} \operatorname{Pr}\left(v(t) \neq w \text { and } X_{t}(w) \neq Y_{t}(w)\right) \\
& +\sum_{w \in V} \operatorname{Pr}\left(v(t)=w \text { and } X_{t+1}(w) \neq Y_{t+1}(w)\right) \\
= & \frac{n-1}{n} h\left(X_{t}, Y_{t}\right)+\frac{1}{n} h\left(X_{t}, Y_{t}\right) \frac{4(k-1) \epsilon}{1-2 \epsilon} \\
\leq & \left(1-\frac{1}{2 n}\right) h\left(X_{t}, Y_{t}\right) .
\end{aligned}
$$

Summarising, we have shown that with probability at least $1-e^{-c \mu_{1}}$ for some positive constant $c$, we have that both of $X_{0}$ and $Y_{0}$ are $\boldsymbol{\epsilon}$-good, both $X$ and $Y$ are $2 \boldsymbol{\epsilon}$-good for 
$t_{0}$ steps and both of $X_{t_{0}}$ and $Y_{t_{0}}$ are $\boldsymbol{\epsilon}$-good. If we run the chain for $t_{0} t^{*}$ steps, where $t^{*}=e^{c \mu_{1} / 2}$ steps then the probability that either chain stops being $2 \boldsymbol{\epsilon}$-good is at most $t^{*} e^{-c \mu_{1}} \leq e^{-c \mu_{1} / 2}$. Conditional on these events, $\mathbf{E}\left(h\left(X_{t_{\delta}}, Y_{t_{\delta}}\right) \leq \delta / 2\right.$ and together with the fact that the variation distance between $X_{t}$ and $Y_{t}$ is monotone non-increasing, this implies (4). (Note that $\delta$ includes the probability that either of $X_{0}, Y_{0}$ are not $2 \boldsymbol{\epsilon}$-good). This completes the proof of Theorem 1.

\subsection{Proof of Corollary 1}

The proof of Theorem 1 shows that if $X, Y \in \mathcal{Q}$ are both $\boldsymbol{\epsilon}$-good then there is a path from $X$ to $Y$ in $\mathcal{Q}$ of length $O(n \log n)$. Since almost all of $\mathcal{Q}$ is $\boldsymbol{\epsilon}$-good, we are done.

\section{Blocked example}

In this section we give an example of an edge colored hypergraph for which there are no Glauber moves.

Lemma 3 Let $k \geq 3$ and let $m, q$ be sufficiently large. Suppose that $\epsilon^{k} \leq \frac{1}{10 k !}$ and $\rho=$ $\frac{\epsilon^{k}}{(q m)^{k-2}}$. Then there exists a hypergraph $H$ with qm vertices and maximum degree $\Delta \in$ $\left[\frac{\epsilon^{k} q m}{2(k-1) !}, \frac{2 \epsilon^{k} q m}{(k-1) !}\right]$ and a coloring with $q$ colors so that there are no Glauber moves.

Proof We first choose a simple $(k-1)$-uniform hypergraph $H_{1}$ with $m$ vertices and $q m$ edges and maximum degree at most $2 k^{4} q$. The existence of such a hypergraph is easy to show via the probabilistic method. Fix some $0<p<1$ and choose each possible edge to include with probability $p$. Let $Z_{1}$ be the number of edges chosen and let $Z_{2}$ be the number of pairs of edges that share two or more vertices. We show is that there is a $p$ such that $\mathbf{E}\left(Z_{1}-Z_{2} \mid D\right) \geq q m$ where $D$ is the event that the maximum degree is at most $2 k^{4} q$. Now $\mathbf{E}\left(Z_{1}\right)=\left(\begin{array}{c}m \\ k-1\end{array}\right) p$ and $\mathbf{E}\left(Z_{2}\right) \leq\left(\begin{array}{c}m \\ k-1\end{array}\right)\left(\begin{array}{c}k-1 \\ 2\end{array}\right)\left(\begin{array}{c}m-2 \\ k-3\end{array}\right) p^{2}$. Putting $p=\frac{k^{4} q}{m\left(\begin{array}{c}k-1 \\ 2\end{array}\right)\left(\begin{array}{c}m-2 \\ k-3\end{array}\right)}$ gives $\mathbf{E}\left(Z_{1}-Z_{2}\right) \geq \frac{k^{4} q(m-1)}{(k-1)^{2}(k-2)^{2}}$. Now the degree of a vertex is $\operatorname{Bin}\left(\left(\begin{array}{c}m-1 \\ k-2\end{array}\right), p\right)$ which has mean $\frac{2 k^{4} q(m-1)}{m(k-1)(k-2)^{2}}$ and so the probability its degree is greater than $2 k^{4} q$ is exponentially small in $q$. Thus $\mathbf{E}\left(Z_{1}-Z_{2} \mid D\right) \geq q m$ and our hypergraph exists.

We build a vertex colored $k$-uniform simple hypergraph $H$ for which the coloring is proper and for which there are no Glauber moves. We choose disjoint sets $V_{1}, V_{2}, \ldots, V_{q}$ of size $m$ and let $V=V_{1} \cup V_{2} \cup \cdots V_{q}$. The vertices in $V_{i}$ are given color $i$. We let $H_{i}=\left(V_{i}, E_{i}\right)$ be a copy of the hypergraph $H_{1}$. Then for each $i$ we define an injective map $f_{i}$ from $V \backslash V_{i} \rightarrow E_{i}$. This is possible as $\left|E_{i}\right|=q m \geq|V|$. Then for each $x \in V_{j}$ and $i \neq j$ we add the edge $F_{x, i}=\{x\} \cup f_{i}(x)$ to $H$. These edges block all Glauber moves. Furthermore, we have (i) $F_{x, i} \cap F_{x, i^{\prime}}=\{x\}$ for $i \neq i^{\prime}$ and (ii) $\left|F_{x, i} \cap F_{y, i}\right| \leq 1$ for $x \neq y$ and (iii) $F_{x, i} \cap F_{y, i}=\emptyset$ for $x \neq y, i \neq j$. Thus the hypergraph created is simple. Denote the set of edges added so far by $F_{1}$ and note that $\left|F_{1}\right| \leq q^{2} m$. 
At the moment the degree of a vertex lies in $\left[q-1,\left(k^{4}+1\right) q-1\right]$. We now add random edges $F_{2}$ so that we have more flexibility with the maximum degree. We only consider edges with at most one vertex in each $V_{i}$ and we add these with probability $\rho$. Now let $A_{1}$ denote the number of pairs of edges in $F_{2}$ that share two or more vertices and let $A_{2}$ denote the number of pairs of edges, one from $F_{1}$ and one from $F_{2}$ that share two or more vertices.

Now $\mathbf{E}\left(\left|F_{2}\right|\right)=\left(\begin{array}{l}q \\ k\end{array}\right) m^{k} \rho$ and our choice of $\rho$ is such that $\mathbf{E}\left(\left|F_{2}\right|\right) \geq 2 \mathbf{E}\left(A_{1}+A_{2}\right)$. Now

$$
\begin{aligned}
& \mathbf{E}\left(A_{1}+A_{2}\right) \leq \\
& \quad\left(\begin{array}{l}
q \\
k
\end{array}\right) m^{k}\left(\begin{array}{l}
k \\
2
\end{array}\right)\left(\begin{array}{l}
q-2 \\
k-2
\end{array}\right) m^{k-2} \rho^{2}+q^{2} m\left(\begin{array}{l}
k \\
2
\end{array}\right)\left(\begin{array}{l}
q-2 \\
k-2
\end{array}\right) m^{k-2} \rho \leq(q m)^{2 k-2} \rho^{2}+k^{2} q^{k} m^{k-1} \rho .
\end{aligned}
$$

Concentration of measure now ensures that $\Delta$ is in the range required.

\section{Open Questions}

(a) Can we replace the lower bound in (2) by $\Delta \geq \Delta_{0}$ ?

(b) Can we replace (3) by $q^{k-1} \geq K \Delta$ for sufficiently large $K$ ?

(c) Can we extend the result to arbitrary $k$-uniform hypergraphs?

Acknowledgement: We thank Mary Cryan for two important observations.

\section{References}

[1] G. R. Brightwell and P. Winkler, Random colorings of a Cayley tree. Contemporary combinatorics, 10:247-276, 2002.

[2] M. Dyer, A. Frieze. Randomly coloring graphs with lower bounds on girth and maximum degree. Random Structures and Algorithms, 23(2):167-179, 2003.

[3] M.E. Dyer, A.M. Frieze and R. Kannan, A random polynomial time algorithm for approximating the volume of convex bodies, Journal of the Association for Computing Machinery 38(1):1-17, 1991.

[4] A.M. Frieze and D. Mubayi, Colouring Simple Hypergraphs.

[5] A.M. Frieze and E. Vigoda, A survey on the use of Markov chains to randomly sample colorings, in Combinatorics, Complexity and Chance, A tribute to Dominic Welsh, (G. Grimmett, C. McDiarmid Eds.) (2007) 53-71

[6] M.R. Jerrum, A very simple algorithm for estimating the number of $k$-colorings of a low-degree graph, Random Structures and Algorithms, 7(2):157-165, 1995. 
[7] M.R. Jerrum, A. Sinclair and E. Vigoda, A polynomial-time approximation algorithm for the permanent of a matrix with non-negative entries, Journal of the Association for Computing Machinery, 51(4):671-697, 2004.

[8] R. Kannan, L. Lovász and M. Simonovits, Random walks and an $O^{*}\left(n^{5}\right)$ volume algorithm for convex bodies, Random Structures and Algorithms, 11(1):1-50, 1997.

[9] L. Lovász and S. Vempala, Simulated Annealing in Convex Bodies and an $O^{*}\left(n^{4}\right)$ Volume Algorithm. In Proceedings of the 44th Annual IEEE Symposium on Foundations of Computer Science (FOCS), 650-659, 2003.

[10] J. Salas and A. Sokal, Absence of phase transition for antiferromagnetic Potts models via the Dobrushin uniqueness theorem, Journal of Statistical Physics, 86(3-4):551-579, 1997.

[11] E. Vigoda, Improved bounds for sampling colorings, Journal of Mathematical Physics, 41(3):1555-1569, 2000. 